Short communication

\title{
Simultaneous low-frequency deep brain stimulation of the substantia nigra pars reticulata and high-frequency stimulation of the subthalamic nucleus to treat levodopa unresponsive freezing of gait in Parkinson's disease: A pilot study
}

\author{
Francesc Valldeoriola*, Esteban Muñoz, Jordi Rumià, Pedro Roldán, Ana Cámara, \\ Yaroslau Compta, María José Martí, Eduardo Tolosa
}

Institut de Neurociències, Hospital Clínic de Barcelona, Universitat de Barcelona, Spain

A R T I C L E I N F O

\section{Keywords:}

Freezing of gait

Parkinson's disease

Substantia nigra reticulata

Low frequency stimulation

\begin{abstract}
A B S T R A C T
Introduction: Experimental studies suggest that low-frequency (LF) (63 Hz) deep brain stimulation (DBS) of the substantia nigra pars reticulata ( $\mathrm{SNr}$ ) could be useful to regulate gait disorders refractory to medical treatment in Parkinson's disease (PD). The SNr neurons could act as high-frequency (HF) pacemakers within locomotor control systems. Currently, no specific therapies can treat gait disorders in PD with insufficient response to dopaminergic treatment.

Objective: To investigate whether LF-SNr-DBS combined with standard HF stimulation of the subthalamic nucleus (STN) is clinically relevant in improving gait disorders that no longer respond to levodopa in PD patients, compared with HF-STN or LF-SNr stimulation alone.

Methods: Patients received LF-SNr or HF-STN stimulation alone or combined (COMB) stimulation of both nuclei (crossover design). The nucleus to be stimulated was randomly assigned and clinical evaluations performed by a blinded examiner after three months follow-up for each. Clinical assessment included the Freezing of Gait questionnaire, Tinetti Balance and Walking Assessing tool, and Unified Parkinson's Disease Rating.

Results: We included six patients (mean age 59.1 years, disease duration 16.1 years). All patients suffered motor fluctuations and dyskinesias. The best results were obtained with COMB in four patients (who preferred and remained with COMB over 3 years of follow-up) and with HF-STN in two patients. SNr stimulation alone did not produce better results than COMB or STN in any patient.

Conclusion: COMB and HF-STN stimulation improved PD-associated gait disorders in this preliminary case series, sustained over time. Further multicenter investigations are required to better explore this therapeutic option.
\end{abstract}

\section{Introduction}

One of the most challenging and unresolved problems in Parkinson's disease (PD) is the treatment of gait disorders unresponsive to dopaminergic medication. Patients suffering from gait disorders are not considered good candidates for subthalamic nucleus deep brain stimulation (STN-DBS) [1]. Currently, there are no specific therapies to treat gait disorders in PD with an insufficient response to dopaminergic treatment. Furthermore, axial symptoms develop during disease progression even in operated patients. Axial symptoms are often one of the main causes of disability [2]. Nonetheless, there is evidence suggesting that low-frequency (LF) stimulation of the ventral area of the STN can improve gait symptoms [3]. The substantia nigra pars reticulata (SNr) is one of the most important output centers of the basal ganglia and is integrated within the locomotor control systems. Neurons within the $\mathrm{SNr}$ may act as high-frequency (HF) pacemakers, disrupting normal behavior downstream in the circuit [4]. Dual HF-STN and LF-SNr stimulation may have beneficial effects in the treatment of gait disorders unresponsive to dopaminergic therapy [5].

The aim of our study is to evaluate whether LF-SNr-DBS $(63 \mathrm{~Hz})$ combined with dorsolateral HF-STN-DBS produces clinically relevant improvements in gait disorders compared with conventional HF-STNor LF-SNr-DBS alone in PD patients.

\footnotetext{
* Corresponding author.

E-mail addresses: fvallde@clinic.cat, fvallde@clinic.ub.es (F. Valldeoriola).
} 


\section{Methods}

This is a prospective, randomized, crossover pilot study with blinded clinical evaluations. The study and informed consent were approved by the Hospital Clinic of Barcelona Ethics Committee (HCB/ 2017/0716). The study conforms to the Declaration of Helsinki. All included patients signed an informed consent.

\subsection{Clinical assessment}

We included six patients diagnosed with PD who were unsuitable candidates for STN-DBS due to the presence of gait disorders with an insufficient response to best dopaminergic treatment. Demographic data and medication was noted, along with video recording and administration of several clinical scales, in best medication "on" and overnight "off" conditions. Clinical assessments included Freezing of Gait (FoG) Questionnaire (self-administered) [6], Unified Parkinson's Disease Rating Scale (UPDRS) in off and on condition, Timed Up-andGo (TUG) test, and Tinetti Balance and Walking Assessing tool [7] (Spanish version). Adverse effects (AEs), and stimulation parameters were recorded at each follow-up visit.

The baseline visit (Supplementary Fig. 1) was performed 3 months before the surgical procedure for candidate screening, administration of scales and informed consent. Stimulation was initiated one week after surgery. Optimization of medication and electrical parameters for each target was done by a movement disorders specialist (FV) during a 3month window following surgery.

Patients were randomized to receive either HF-STN-DBS or LF-SNrDBS and were evaluated after a 3-month follow-up period. After 3 months, all patients crossed over to COMB stimulation, using optimal settings obtained in the previous phases, and also evaluated after 3 months. All assessment scales were evaluated by a rater (EM) blinded to the stimulation target, parameters, and medication.

\subsection{Statistical analysis}

Given the small number of patients in this study, only descriptive statistics were used.

\subsection{Surgical procedure}

Bilateral STN-DBS was carried out through stereotaxy and conscious sedation. A stereotactic planning software (iPlan from BrainLab AG, Munich, Germany) was used to determine STN coordinates, based on direct targeting in 3 T MRI. Intraoperative microrecordings and stimulation (Neuroset, Neurostar GmbH, Tübingen Germany) were used to confirm an adequate location before implanting the electrode (ModelDB-2201-45, Boston Scientific, Valencia, CA). The interface between the STN and SNr was determined based on microelectrode recording. The macroelectrode was advanced $3 \mathrm{~mm}$ below the identified ventral border of the STN. Intraoperative stimulation was also carried out at the most ventral border of the dorsolateral STN. This procedure enabled us to stimulate the $\mathrm{SNr}$ as well as the dorsolateral STN. Finally, a neurostimulator (Vercise DB-1110-C, Boston Scientific, Valencia, CA) was implanted. The final lead location was determined by cranial MRI $24 \mathrm{~h}$ after surgery.

\subsection{Stimulation parameters}

The pulse width was set to $60 \mu$ in all patients and stimulation amplitude was adjusted to maximize the patient's clinical benefits via monopolar review. The objective of the dual stimulation was to use a frequency of $126 \mathrm{~Hz}$ (HF) for STN-DBS and $63 \mathrm{~Hz}$ (LF) for SNr-DBS in both hemispheres, for a total of four different stimulation areas. The neurostimulator used meant that if more than two areas were ON, all areas should have the same rate. To resolve this, we decided to double the rate by stimulating one area at $63 \mathrm{~Hz}$ targeting both the STN and $\mathrm{SNr}$, and then creating another area at $63 \mathrm{~Hz}$ targeting the STN only, so the frequency of pulses would be double at the STN (i.e. $126 \mathrm{~Hz}$ ). We calculated the desired amplitude in the area where the STN and SNr were stimulated simultaneously, taking advantage of multiple, independent current-controlled technology, which allows different percentages of current to be delivered independently. Due to a safety feature of the device preventing the temporal overlap of stimulation pulses to different areas, the exact temporal fidelity between any two pulses in the HF stimulation cannot be guaranteed to be fixed. Nonetheless, this safety feature does not affect the frequency of stimulation delivered.

\section{Results}

\subsection{Demographic and clinical data}

We included six patients (five male), with a mean age of 59.1 (range 43-70) years, mean disease duration of $16.1(10-20)$ years, and mean "on" freezing duration of 7.3 (3-15) years. Two of the patients had early onset PD (starting at ages 43 and 37), with both patients exhibiting mutation in the gene encoding the 'Parkin' protein. The levodopa equivalent dose (LEDD) was $1250 \pm 427$ (550-1850) mg/day. All patients suffered motor fluctuations and troublesome peak dose dyskinesias. Details of the contacts and stereotactic coordinates for each patient can be found in Supplementary Table 1.

\subsection{Motor assessment}

Self-administered FoG questionnaire. Evaluation of gait in the worst condition indicated that at baseline, two patients were unable to walk and four required a cane; after treatment, gait improved (i.e. almost normal gait or no help needed) in four patients after COMB stimulation, two patients after HF-STN-DBS, and one patient after LF-SNrDBS.

All patients presented the maximal score for FoG at baseline (Fig. 1A), indicating that FoG is always present when walking. At final follow-up, the prevalence of FoG in COMB patients was "never" (one patient), "very rarely or once per month" (one patient), "rarely or once per week" (one patient), or "continuous" (three patients). Unexpectedly, HF-STN-DBS patients presented with a FoG prevalence of "never" (one patient), "very rarely" (one patient), or "continuous" (four patients). Duration of freezing (Fig. 1B) was reduced in five patients after COMB stimulation and two patients after HF-STN-DBS. Start hesitation (Fig. 1C) did not occur in two patients with COMB stimulation and one patient with HF-STN-DBS, and improved in four patients after COMB stimulation and four patients after HF-STN-DBS. Duration of freezing while turning (Fig. 1D) was clearly reduced in five patients with COMB stimulation, three with HF-STN-DBS, and five with LF-SNrDBS. Freezing when turning (Fig. 1D) disappeared in one patient after COMB stimulation, and in one patient after HF-STN-DBS.

An improvement in activities of the daily living was noted after all interventions (Table 1). All six patients had a severe gait disorder at baseline (as per the inclusion criteria); after intervention, improvement (no, or slight problems) was observed in five patients after COMB, two after HF-STN-DBS, and three patients after LF-SNr-DBS.

Tinetti Balance and Walking Assessing tool. All three stimulation paradigms resulted in benefits in equilibrium, gait, and total scores when compared to baseline, with COMB stimulation toward slightly increased benefit over either STN or $\mathrm{SNr}$ stimulation alone (Supplementary Figs. 2a and b).

UPDRS part II and III. Scores of the UPDRS II and III, axial items (28, 29, 30, 31) of the UPDRS, and item 29 (gait), "on" and "off" medication, at baseline and at the end of each follow-up assessment in the different targets are shown in Table 1.

Medication. LEDD reduction was $63 \%$ for HF-STN-DBS alone, $43 \%$ 


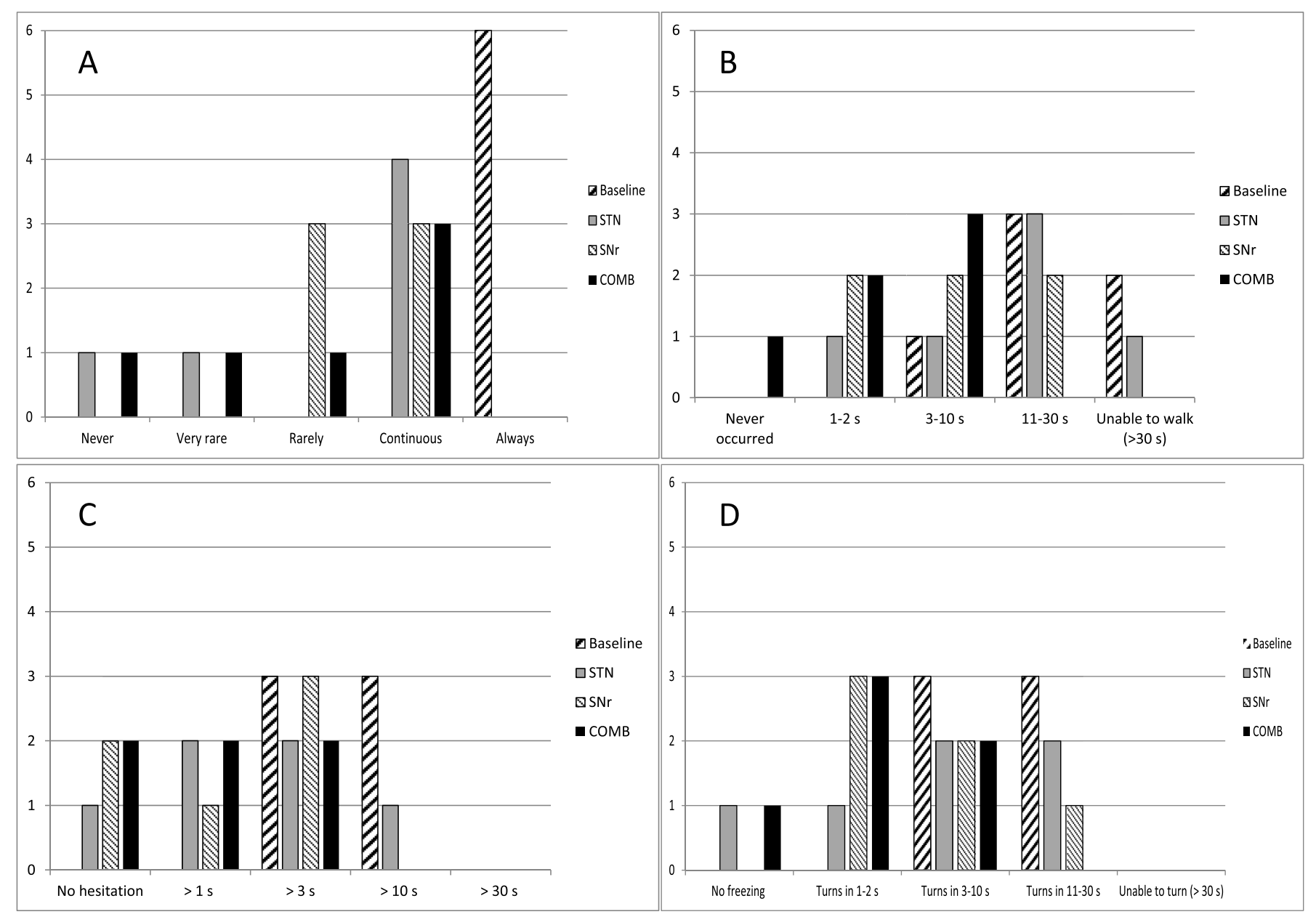

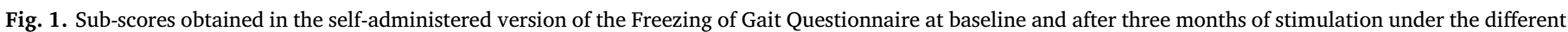

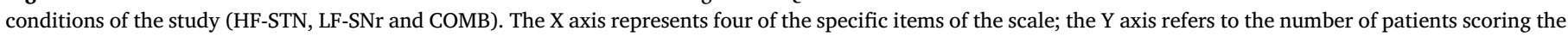
specific item.

A) Freezing of gait: Do you feel that your feet get glued to the floor while walking, making a turn or when trying to initiate walking (freezing)?

B) Duration of freezing: How long is your longest freezing episode?

C) Start hesitation: How long is your typical start hesitation episode (freezing when initiating the first step)?

D) Freezing while turning: How long is your typical turning hesitation (freezing when turning)?

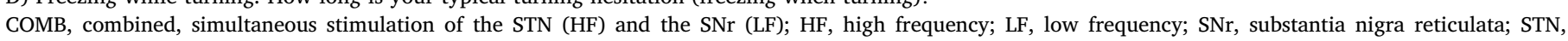
subthalamic nucleus.

for LF-SNr-DBS alone, and 53\% for COMB stimulation.

\subsection{Stimulation parameters}

For HF-STN-DBS alone, the mean amplitude was $2.9 \pm 0.5$ (range 2-3.5) $\mathrm{mA}$ in the right hemisphere and $2.9 \pm 0.6(2-3.6) \mathrm{mA}$ in the left. For LF-SNr-DBS alone, amplitude was $4 \pm 1$ (3-5.5) $\mathrm{mA}$ in the right hemisphere and $4.8 \pm 0.5(4-5.2) \mathrm{mA}$ in the left. For HF-STNDBS, monopolar setup was used in all patients in both sides, using dorsal contacts. For LF-SNr-DBS, we programmed a monopolar setup in two patients and bipolar configuration in the other four patients, using the most ventral contacts in both sides.

Four out of the six patients preferred to maintain dual stimulation after the study (COMB). Final parameters for HF-STN-DBS were $3 \pm 1$ (2-4) $\mathrm{mA}$ on the right side and $2.6 \pm 0.9(2-4) \mathrm{mA}$ on the left. For LFSNr-DBS, right parameters were $3.6 \pm 1.4(2.5-5.6) \mathrm{mA}$ and left $2.3 \pm 2(0.8-5) \mathrm{mA}$. The two remaining patients decided to receive HF-STN-DBS alone.

\subsection{Adverse effects}

No intraoperative AEs were observed. Postoperative AEs included confused state (one patient), which improved after two weeks and resolved in 1 month. Transitory AEs included blurred vision (two LF-SNrDBS patients), and muscular twitching (two HF-STN-DBS patients), both corrected after reducing the stimulation amplitude. One patient developed depressive symptoms after STN stimulation, which did not improve after switching OFF the stimulator for one week and which were attributed to dopamine agonist withdrawal.

\section{Discussion}

To our knowledge, this pilot study is the first to study the effects of simultaneous DBS in both the STN and SNr at different frequencies in PD (COMB stimulation). New stimulation systems allow independent stimulation with multiple frequencies, enabling easy evaluation of the impact of stimulating different targets at different frequencies. Our study was designed to specifically analyze the possible efficacy of such approach in improving levodopa-unresponsive FoG. Our results show that COMB produced a sustained improvement in PD-associated gait 
Table 1

Scores of the UPDRS II and III, axial items $(28,29,30,31)$ of the UPDRS, and gait (item 29), both "off" and "on" medication, at baseline and at the end of each follow-up assessment (3 months) in the different targets (HF-STN, LF-SNr and $\mathrm{COMB}$ ).

\begin{tabular}{lllllllll}
\hline \multirow{2}{*}{ Patient } & ADL & \multicolumn{2}{c}{ UPDRS III } & \multicolumn{2}{c}{ UPDRS axial items } & \multicolumn{2}{c}{ Gait (item 29) } \\
\cline { 2 - 9 } & OFF & ON & OFF & ON & OFF & ON & OFF & ON \\
\hline \multicolumn{2}{l}{ Preoperative } & & & & & & & \\
1 & 20 & 19 & 31 & 12 & 7 & 6 & 3 & 3 \\
2 & 31 & 22 & 37 & 28 & 10 & 5 & 3 & 3 \\
3 & 15 & 6 & 33 & 18 & 7 & 6 & 4 & 3 \\
4 & 20 & 9 & 28 & 18 & 8 & 6 & 3 & 3 \\
5 & 32 & 19 & 34 & 19 & 10 & 7 & 4 & 2 \\
6 & 12 & 8 & 23 & 8 & 6 & 5 & 3 & 3 \\
\hline
\end{tabular}

\begin{tabular}{lllllllll}
\hline STN & & & & & & & & \\
1 & 17 & 6 & 10 & 4 & 4 & 2 & 1 & 0 \\
2 & 15 & 10 & 20 & 14 & 6 & 4 & 2 & 1 \\
3 & 9 & 9 & 16 & 15 & 3 & 3 & 1 & 0 \\
4 & 8 & 6 & 16 & 14 & 3 & 3 & 2 & 1 \\
5 & 20 & 11 & 24 & 15 & 5 & 2 & 3 & 2 \\
6 & 13 & 4 & 21 & 5 & 6 & 3 & 3 & 2 \\
\hline
\end{tabular}

\begin{tabular}{lllllllll}
\hline SNr & & & & & & & & \\
1 & 11 & 5 & 22 & 3 & 3 & 2 & 1 & 0 \\
2 & 29 & 22 & 40 & 27 & 10 & 5 & 2 & 2 \\
3 & 13 & 9 & 30 & 15 & 7 & 2 & 2 & 1 \\
4 & 8 & 8 & 23 & 16 & 4 & 3 & 2 & 1 \\
5 & 30 & 18 & 29 & 19 & 7 & 5 & 3 & 2 \\
6 & 13 & 4 & 20 & 8 & 5 & 3 & 3 & 2 \\
\hline
\end{tabular}

\begin{tabular}{lllllllll}
\hline COMB & & & & & & & & \\
1 & 14 & 6 & 10 & 4 & 4 & 2 & 1 & 0 \\
2 & 27 & 19 & 28 & 27 & 6 & 4 & 1 & 1 \\
3 & 5 & 3 & 22 & 22 & 2 & 2 & 1 & 0 \\
4 & 3 & 2 & 10 & 8 & 2 & 1 & 1 & 0 \\
5 & 30 & 18 & 20 & 13 & 6 & 2 & 3 & 2 \\
6 & 14 & 5 & 18 & 5 & 3 & 2 & 2 & 2
\end{tabular}

ADL, activities of daily living; COMB, combined, simultaneous stimulation of the STN (HF) and the SNr (LF); HF, high frequency; LF, low frequency; SNr, substantia nigra reticulata; STN, subthalamic nucleus.

\section{disorders.}

The $\mathrm{SNr}$ is a major output nucleus of the basal ganglia, sending projections to the brainstem and thalamus. $\mathrm{SNr}$ projections to the pontine tegmental region are involved in posture control [8]. The first data to suggest that LF stimulation of the STN reduces the number of freezing episodes in PD patients compared to HF $(130 \mathrm{~Hz})$ stimulation came from Moreau et al. [3], who concluded that the frequency of STNDBS could be changed to $60 \mathrm{~Hz}$ at high voltage to improve axial symptoms in PD patients that develop severe gait disorders. This study also found that FoG worsens when the voltage was increased at LF stimulation. The change in the clinical response following variations in the stimulation frequency is controversial.

One study has shown improvement or non-worsening of FoG using frequencies lower than $100 \mathrm{~Hz}$ [9]; others have demonstrated transient [10] absence of effect of LF stimulation [11]. We tested LF stimulation of the STN using the most ventral contacts in several patients who developed gait problems during disease progression after chronic DBS, and obtained clear improvements in the number of freezing episodes and step length in some of them.

A crossover, double-blind, randomized controlled trial using STNvs. dual stimulation at HF, using interleaved pulses in 12 PD patients, showed that HF, interleaved, combined stimulation improved the Freezing of Gait Assessment Course without changes in postural control [5]. In a recent review [12], it was found that overall LF stimulation seemed to be consistently effective in patients with FoG previously treated with standard HF stimulation.

Four out of six patients achieved better global results with COMB stimulation than HF-STN- or LF-SNr-DBS alone. These patients preferred and remained with COMB over three years follow-up. Our results seem to be more promising than those obtained with combined stimulation at $130 \mathrm{~Hz}$ [5]. LF-SNr-DBS alone resulted in a relative improvement in FoG, but the global antiparkinsonian effect was lower than that achieved with HF-STN-DBS. Blurred (double) vision was a limitation to increasing intensity in two patients stimulated in the SNr. Behavioral changes have been reported when stimulating ventral and medial areas of the STN. We did not observe cognitive changes after stimulation in any target. One patient developed depressive symptoms after STN stimulation that were attributed to dopamine agonist withdrawal. Similarly, in a study showing the effects of STN and SNr stimulation at high frequencies no serious behavioral or psychiatric adverse effects were found [5].

Some patients also obtained remarkable benefit in the amelioration of "on" freezing with isolated HF-STN stimulation - which may be one of the more remarkable finding of our study. The beneficial effects on gait observed with COMB stimulation and isolated STN stimulation were maintained for three years after the intervention, ruling out the possibility of any placebo effect. Although improvement of FoG is challenging in patients stimulated in the STN as the disease progresses, this finding could be considered in the future for patients with medically-untreatable FoG with otherwise good response to levodopa in other parkinsonian signs. The low number of cases included in this preliminary pilot study makes it absolutely necessary to develop further multicentric investigations to obtain the sufficient number of patients and obtain more consistent conclusions regarding this challenging topic.

\section{Funding}

This study was funded by a grant from Boston Scientific (Valencia, CA, USA).

\section{Declarations of interest}

Francesc Valldeoriola received honoraria for advice and lectures from Boston Scientific.

\section{Appendix A. Supplementary data}

Supplementary data related to this article can be found at https:// doi.org/10.1016/j.parkreldis.2018.09.008.

\section{References}

[1] J.M. Bronstein, M. Tagliati, R.L. Alterman, A. M Lozano, J. Volkmann, A. Stefani, F.B. Horak, M.S. Okun, K.D. Foote, P. Krack, R. Pahwa, J. M Henderson, M.I. Hariz, R.A. Bakay, A. Rezai, W.J. Jr Marks, E. Moro, J. L Vitek, F.M. Weaver, R. E Gross, M. R DeLong, Deep brain stimulation for Parkinson disease: an expert consensus and review of key issues, Arch. Neurol. 68 (2011) 165.

[2] M. Zibetti, A. Merola, L. Rizzi, V. Ricchi, S. Angrisano, C. Azzaro, C.A. Artusi, N. Arduino, A. Marchisio, M. Lanotte, M. Rizzone, L. Lopiano, Beyond nine years of continuous subthalamic nucleus deep brain stimulation in Parkinson's disease, Mov. Disord. 26 (2011) 2327-2334.

[3] C. Moreau, L. Defebvre, A. Destee, S. Bleuse, F. Clement, J.L. Blatt, P. Krystkowiak, D. Devos, STN-DBS frequency effects on freezing of gait in advanced Parkinson disease, Neurology 71 (2008) 80-84.

[4] J.W. Barter, S. Castro, R. Sukharnikova, M.A. Rossi, H.H. Yin, The role of the substantia nigra in posture control, Eur. J. Neurosci. 39 (2014) 1465-1473.

[5] D. Weiss, M. Walach, C. Meisner, M. Fritz, M. Scholten, S. Breit, C. Plewnia B. Bender, A. Gharabaghi, T. Wächter, R. Krüger, Nigral stimulation for resistant axial motor impairment in Parkinson's disease? A randomized controlled trial, Brain 136 (2013) 2098-2108.

[6] M. Nilsson, G.-M. Hariz, K. Wictorin, M. Miller, L. Forsgren, P. Hagell, Development and testing of a self-administered version of the freezing of gait questionnaire, BMC Neurol. 10 (2010) 85.

[7] D.A. Kegelmeyer, A.D. Kloos, K.M. Thomas, S.K. Kostyk, Reliability and validity of the Tinetti mobility test for individuals with Parkinson disease, Phys. Ther. 87 (2007) 1369-1378.

[8] K. Takakusaki, N. Tomita, M. Yano, Substrates for normal gait and pathophysiology 
of gait disturbances with respect to the basal ganglia dysfunction, J. Neurol. 255 (2008) 19-29.

[9] A. Fasano, A.M. Lozano, The FM/AM world is shaping the future of deep brain stimulation, Mov. Disord. 29 (2014) 161-163.

[10] V. Ricchi, M. Zibetti, S. Angrisano, A. Merola, N. Arduino, C.A. Artusi, M. Rizzone, L. Lopiano, M. Lanotte, Transient effects of $80 \mathrm{~Hz}$ stimulation on gait in STN DBS treated PD patients: a 15 months follow-up study, Brain Stimul 5 (2012) 388-392.
[11] C. Sidiropoulos, R. Walsh, C. Meaney, Y.Y. Poon, M. Fallis, E. Moro, Low-frequency subthalamic nucleus deep brain stimulation for axial symptoms in advanced Parkinson's disease, J. Neurol. 260 (2013) 2306-2311.

[12] T. Xie, M. Padmanaban, L. Bloom, E. MacCracken, B. Bertacchi, A. Dachman, P. Warnke, Effect of low versus high frequency stimulation on freezing of gait and other axial symptoms in Parkinson patients with bilateral STN DBS: a mini-review, Transl. Neurodegener. 6 (2017) 13. 